\title{
Recurrence Pattern and Complication Rate of Allergic Fungal Sinusitis: A 10-Year Tertiary Center Experience
}

\author{
Yazeed Alghonaim, ${ }^{1,2}$ Abdulrhman Alfayez, ${ }^{1,2}$ Riyadh Alhedaithy, ${ }^{1}$ Abdullah Alsheikh, ${ }^{3}$ \\ and Malak Almalki (iD ${ }^{4}$ \\ ${ }^{1}$ ORL-H↔N Surgery Department, King Abdulaziz Medical City, Riyadh, Saudi Arabia \\ ${ }^{2}$ ORL-HeN Surgery, King Saud Bin Abdulaziz University for Health Sciences, Riyadh, Saudi Arabia \\ ${ }^{3}$ ORL-HeN Surgery Department, King Abdulaziz University Hospital, Riyadh, Saudi Arabia \\ ${ }^{4}$ King Saud Bin Abdulaziz University for Health Sciences, Riyadh, Saudi Arabia \\ Correspondence should be addressed to Malak Almalki; almalki130@ksau-hs.edu.sa
}

Received 22 June 2020; Revised 25 November 2020; Accepted 12 December 2020; Published 18 December 2020

Academic Editor: Roberto O. Dantas

Copyright (@) 2020 Yazeed Alghonaim et al. This is an open access article distributed under the Creative Commons Attribution License, which permits unrestricted use, distribution, and reproduction in any medium, provided the original work is properly cited.

\begin{abstract}
Background. Allergic fungal rhinosinusitis is a noninvasive form of highly recurrent chronic rhinosinusitis. Despite the advancement in medical and surgical strategies, recurrence in AFRS in general poses another challenging problem with reported incidence that eventually can reach more than $60 \%$. Recognition and understanding the pattern of disease recurrence will lead to greater understanding of the disease response in our population. Method. A retrospective cohort study was performed in King Abdulaziz Medical City in Riyadh, Saudi Arabia. All patients diagnosed with chronic rhinosinusitis and underwent functional endoscopic sinus surgery from the period of January 2006 to December 2016 were reviewed. Results. 28 patients were found to have AFRS based on clinical, radiological, and microscopic examination suggestive of allergic fungal rhinosinusitis. Among these patients, $53 \%$ of them were female and $46 \%$ were male. The age ranged from 13 to 55 years, with a mean age of 31.57 years. $28.57 \%$ of the patients presented with recurrent allergic fungal sinusitis. The duration between the surgery and symptoms recurrence was around one year. Male and female patients had similar recurrence rate (50\%). At first visit, $95 \%$ of the patients with nonrecurrent disease presented with nasal obstruction compared to $87.5 \%$ of the patients with recurrent disease. On the other hand, patients with recurrent disease had more nasal discharge (87.5\%), postnasal drip (37.5\%), facial pressure/pain (50\%), headache (50\%), nasal polyposis $(87.5 \%)$, hypertrophy of inferior turbinate $(37.5 \%)$, and proptosis $(12.5 \%)$. Nasal obstruction (87.5\%) and nasal polyps (87.5\%) were the most common presenting symptoms for the disease recurrence. The pattern of disease recurrence in the previously unilateral disease was $18 \%$ ipsilateral and $27 \%$ bilateral. For the patients who had bilateral disease formerly, $17 \%(n=3)$ of them had recurrent bilateral disease. Conclusion. Allergic fungal rhinosinusitis is a distinct clinical entity. A high recurrence rate is a pathognomonic feature of the disease, despite all the development in medical and surgical trials. This study demonstrated that recurrence rate is lower in our population. However, more studies with a greater number of patients are needed in the future to clearly recognize the pattern of recurrence in patients with AFRS.
\end{abstract}

\section{Introduction}

Allergic fungal rhinosinusitis (AFRS) is a noninvasive form of highly recurrent chronic rhinosinusitis. It can be distinguished clinically, histopathologically, and prognostically from the other forms of chronic fungal rhinosinusitis. There are three recognized forms of invasive fungal sinusitis (acute necrotizing, chronic invasive, and granulomatous invasive), in addition to two noninvasive forms (fungal ball and allergic fungal) [1]. Allergic mucin and polyps are the hallmark of the disease [2]. The prevalence of AFRS appears to vary by geographical region. The majority of the reported cases were located in high-temperature regions where the humidity is high relatively [3]. The clinical presentation of AFRS patients includes nasal discharge which generally has a thick, greenish-brown mucoid appearance with a "peanut butter"- 
like consistency along with green to black rubbery nasal plugs [4].

Diagnosis criteria of AFRS were established by Bent and Kuhn which contain major and minor criteria. Major criteria include type I hypersensitivity, nasal polyposis, characteristics CT scan findings, presence of eosinophilic mucin, and positive fungal smear. The minor criteria include young individuals, coexistence asthma, unilateral predominance, radiographic bone erosion, fungal culture, Charcot-Leyden crystals, and serum eosinophilia [5].

There were multiple research studies done on AFRS locally and worldwide, and few of them questioned the recurrence rate and pattern specifically in the initially involved and noninvolved sinuses. In addition, despite the advancement in medical and surgical strategies, recurrence in AFRS in general poses another challenging problem with reported incidence that eventually can reach more than $60 \%$ $[6,7]$.

Our aim in this research is to shed light on the rate and pattern of recurrence in the sinuses and whether none involved side will be involved later on during the recurrence phase since it is considered as one of the minor criteria for the diagnosis of AFRS.

\section{Methods}

After obtaining the ethical approval, a retrospective cohort study was performed in King Abdulaziz Medical City in Riyadh, Saudi Arabia. Charts of all patients diagnosed with chronic rhinosinusitis (CRS) and underwent functional endoscopic sinus surgery (FESS) from the period of January 2006 to December 2016 were reviewed. Patients fulfilling the Bent and Kuhn criteria for the diagnosis of allergic fungal sinusitis (AFS) were included [5]. Patients without surgical intervention or who were lost follow-up in the given period were excluded. A data collection sheet designed by the researchers included different variables such as demographic information, clinical presentations, site and side of sinuses involvement, course of management, and recurrence rate and pattern. The main outcome variable was the recurrence of allergic fungal sinusitis (AFS). Data analysis was done using SPSS. Descriptive statistics were presented as percentages and frequencies and numerical variables interpreted by means and standard deviations. Inferential statistics were calculated using the chi-square test. A $p$ value less than 0.05 was considered significant.

\section{Results}

3.1. Patient Characteristics at First Visit. 28 patients were found to have AFRS based on clinical, radiological, and microscopic examination suggestive of allergic fungal rhinosinusitis. Among these patients, 53\% of them were female and $46 \%$ were male. The age ranged from 13 to 55 years, with mean age was 31.57 years. The most common clinical presentation was nasal obstruction (92\%), followed by nasal polyps (82\%), deviated nasal septum (57\%), nasal discharge (50\%), and headache (42\%). Other clinical presentations include facial pressure/pain (28\%), postnasal drip (21\%), hypertrophy of inferior turbinate $(21 \%)$, and proptosis and decreased vision (3\%). For the past medical history, $46 \%$ of the patients had asthma, $17 \%$ had allergic rhinitis, and $7 \%$ had aspirin intolerance. $32 \%$ of the patients underwent nasal surgical intervention previously. The majority of the patients had FESS (21\%), and the remaining had septoplasty (10\%). Table 1 summarizes demographic data, clinical presentation, past medical history, and past surgical history at first visit.

3.2. Patient Characteristics in Recurrent vs Nonrecurrent Disease at First Visit. At first visit, 95\% of the patients who did not have recurrent disease presented with nasal obstruction compared to $87.5 \%$ of the patients with recurrent disease. Nasal discharge was more common in patients with recurrent disease $(87.5 \%)$ compared to patients with nonrecurrent disease (35\%). Patients with recurrent disease had more postnasal drip (37.5\%), facial pressure/pain (50\%), headache $(50 \%)$, nasal polyposis $(87.5 \%)$, hypertrophy of inferior turbinate $(37.5 \%)$, and proptosis $(12.5 \%)$. Patients with nonrecurrent disease had more deviated nasal septum (65\%) and decreased vision (5\%). For the past medical history, patients with recurrent disease had higher incidence of asthma (62.5\%) and allergic rhinitis (25\%) compared to patients with nonrecurrent disease. On the other hand, aspirin intolerance was only found in patients with nonrecurrent disease (10\%). Past nasal surgical intervention was done in $37.5 \%$ of the patients with recurrent disease, and $30 \%$ of patients with nonrecurrent disease. Table 2 shows the comparison between the patients who got recurrence and patients who did not in clinical presentation, past medical history, and past surgical history at first visit.

3.3. Radiological Characteristics of the First CT Scan. All of the 28 patients had heterogenous opacifications. Maxillary sinus was the most common sinus involved (96\%). Anterior ethmoid was involved in $75 \%$ of the cases, compared to posterior ethmoid which was evident in $64 \%$ of the cases. Additionally, sphenoid sinus was involved in $75 \%$ of the cases, and frontal sinus was involved in $71 \%$ of the cases. Moreover, $57 \%$ of the patients had deviated nasal septum, and $25 \%$ had concha bullosa. For the bony defect, 2 patients had infraorbital defect, and only 1 patient had intracranial defect. Table 3 demonstrates the radiological characteristics of the first CT scan.

3.4. Surgical Side and Postoperative Management. Among the 28 patients, 11 patients $(39 \%)$ underwent unilateral sinus surgery, and 17 patients (60\%) underwent bilateral sinus surgery. 21 patients (79\%) had mucin intraoperatively. Nasal steroid spray was used postoperatively by 17 patients $(57 \%)$ while 5 patients $(17.86 \%)$ used nasal steroid irrigation. In addition, 12 patients (43.86\%) used oral steroid as well. Table 4 demonstrates surgery side and its post-op management results.

3.5. Recurrence Rate and Characteristics. $28.57 \%(n=8)$ of the patients presented with recurrent allergic fungal sinusitis. The duration between the surgery and symptoms 
TABLE 1: Characteristics of patients included in the study.

\begin{tabular}{|c|c|c|c|}
\hline Demographics & & $N$ & $\%$ \\
\hline \multirow{2}{*}{ Gender } & Male & 13 & 46.43 \\
\hline & Female & 15 & 53.57 \\
\hline \multirow[t]{5}{*}{ Age } & & $13-55$ & 31.57 (mean) \\
\hline & Nasal obstruction & 26 & 92.86 \\
\hline & Nasal discharge & 14 & 50 \\
\hline & PND & 6 & 21.43 \\
\hline & Facial pressure/pain & 8 & 28.57 \\
\hline \multirow[t]{6}{*}{ Clinical presentation } & Headache & 12 & 42.86 \\
\hline & Nasal polyp & 23 & 82.14 \\
\hline & DNS & 16 & 57.14 \\
\hline & HIT & 6 & 21.43 \\
\hline & Proptosis & 1 & 3.57 \\
\hline & Decreased vision & 1 & 3.57 \\
\hline \multirow{4}{*}{ Past medical history } & Asthma & 13 & 46.43 \\
\hline & Allergic rhinitis & 5 & 17.86 \\
\hline & Aspirin intolerance & 2 & 7.14 \\
\hline & & 9 & 32.14 \\
\hline \multirow[t]{2}{*}{ Past surgical history } & FESS & 6 & 21.43 \\
\hline & Septoplasty & 3 & 10.71 \\
\hline
\end{tabular}

TABLE 2: Comparison between patients with recurrence and patients without recurrence.

\begin{tabular}{|c|c|c|c|c|c|}
\hline & & $N$ (nonrecurrent patients) & $\%$ & $N$ (recurrent patients) & $\%$ \\
\hline \multirow{11}{*}{ Clinical presentation } & & 20 & & 8 & \\
\hline & Nasal obstruction & 19 & 95 & 7 & 87.5 \\
\hline & Nasal discharge & 7 & 35 & 7 & 87.5 \\
\hline & PND & 3 & 15 & 3 & 37.5 \\
\hline & Facial pressure/pain & 4 & 20 & 4 & 50 \\
\hline & Headache & 8 & 40 & 4 & 50 \\
\hline & Nasal polyp & 16 & 80 & 7 & 87.5 \\
\hline & DNS & 13 & 65 & 3 & 37.5 \\
\hline & HIT & 3 & 15 & 3 & 37.5 \\
\hline & Proptosis & 0 & 0 & 1 & 12.5 \\
\hline & Decreased vision & 1 & 5 & 0 & 0 \\
\hline \multirow{3}{*}{ Past medical history } & Asthma & 8 & 40 & 5 & 62.5 \\
\hline & Allergic rhinitis & 3 & 15 & 2 & 25 \\
\hline & Aspirin intolerance & 2 & 10 & 0 & 0 \\
\hline Past surgical history & & 6 & 30 & 3 & 37.5 \\
\hline
\end{tabular}

recurrence was around one year, but it ranged from 2 months to 2 years. Male and female had similar recurrence rate $(50 \%, n=4)$. Most of the patients presented with nasal obstruction $(87.5 \%, n=7)$ and nasal polyps $(87.5 \%, n=7)$. In addition, half of the patients $(50 \%, n=4)$ presented with nasal discharge, facial pain/pressure, and headache.

Table 5 summarizes the recurrence rate and characteristics.

3.6. Recurrence Side Pattern. The pattern of disease recurrence in the previously unilateral disease was $18 \%(n=2)$ ipsilateral and $27 \%(n=3)$ bilateral. For the patients who had bilateral disease formerly, $17 \%(n=3)$ of them had recurrent bilateral disease. In summary, among the 8 patients with recurrent disease, 2 patients (25\%) presented with unilateral disease and 6 patients (75\%) presented with bilateral disease. Table 6 demonstrates disease recurrence pattern side.

\section{Discussion}

In this study, 28 patients were diagnosed to have AFRS depending on clinical, radiological, and microscopic examination suggestive of allergic fungal rhinosinusitis. Our aim was to study the pattern of recurrence of AFRS although we studied multiple aspects including demographic information, clinical presentations, site and side of sinuses involvement, and course of management.

The mean age in our study was 31.57 years with a range of 13-55 years. This is quite similar to a study conducted in India in which the mean age was 28.4 years with a range of 18-48 years [8]. In contrast, in the USA in 2008, the mean 
TABle 3: Radiological characteristics of the first CT scan.

\begin{tabular}{|c|c|c|c|c|}
\hline \multicolumn{3}{|l|}{ Radiological characteristics } & \multirow{2}{*}{$\begin{array}{l}N \\
28\end{array}$} & \multirow{2}{*}{$\begin{array}{c}\% \\
100\end{array}$} \\
\hline \multirow{25}{*}{ Heterogeneous opacification } & \multirow{5}{*}{ Maxillary } & & & \\
\hline & & & 27 & 96.43 \\
\hline & & Right & 10 & 35.71 \\
\hline & & Left & 9 & 32.14 \\
\hline & & Bilateral & 8 & 28.57 \\
\hline & \multirow{5}{*}{ Anterior ethmoid } & & 21 & 75 \\
\hline & & Right & 7 & 25 \\
\hline & & Left & 7 & 25 \\
\hline & & Bilateral & 7 & 25 \\
\hline & & & 18 & 64.29 \\
\hline & \multirow{3}{*}{ Posterior ethmoid } & Right & 7 & 25 \\
\hline & & Left & 4 & 14.29 \\
\hline & & Bilateral & 7 & 25 \\
\hline & \multirow{5}{*}{ Frontal } & & 20 & 71.43 \\
\hline & & Right & 5 & 17.86 \\
\hline & & Left & 7 & 25 \\
\hline & & Bilateral & 8 & 28.57 \\
\hline & & & 21 & 75 \\
\hline & \multirow{4}{*}{ Sphenoid } & Right & 6 & 21.43 \\
\hline & & Left & 6 & 21.43 \\
\hline & & Bilateral & 9 & 32.14 \\
\hline & & & 7 & 25 \\
\hline & \multirow{3}{*}{ Concha bullosa } & Right & 5 & 17.86 \\
\hline & & Left & 2 & 7.14 \\
\hline & & Bilateral & 0 & 0 \\
\hline \multirow[t]{2}{*}{ DNS } & & & 16 & 57.14 \\
\hline & & & 2 & 7.14 \\
\hline \multirow[t]{2}{*}{ Bony defect } & Infraorbital & & 2 & 7.14 \\
\hline & Intracranial & & 1 & 3.57 \\
\hline
\end{tabular}

TABLE 4: Surgery side and post-op management results.

\begin{tabular}{lccc}
\hline & & $N$ & \\
\hline & & & \\
& & 11 & 39.29 \\
Surgery & Unilateral & 17 & 60.71 \\
& Bilateral & 21 & \\
& Mucin & & \\
Post-op management & & 57.14 \\
& Nasal steroid spray & 5 & 17.86 \\
& Nasal steroid irrigation & 12 & 43.86 \\
\hline
\end{tabular}

age was found to be on the higher side, being 45 years with a range of $18-88$ years [9].

The most common signs and symptoms in the first visit were as follows: nasal obstruction $26(92.86 \%)$, nasal polyps $23(82.14 \%)$, and deviated nasal septum 16 (57.14\%). This was similar to a study conducted in King Abdulaziz University Hospital, Riyadh, Kingdom of Saudi Arabia, in which the most common signs and symptoms were as follows: nasal obstruction $24(96 \%)$, nasal polyps $22(88 \%)$, and deviated nasal septum 17 (68\%) [10].

We assessed the past medical and surgical history in all the patients which showed that bronchial asthma is the most common medical history (46.43\%) followed by allergic disorders (17.86\%). Past nasal surgeries were found in $32.14 \%$ of patients. In contrast, the study which was done in India showed that previous nasal surgeries were seen in $20 \%$ of cases followed by bronchial asthma (14.2\%) and allergic disorders (11.42\%) [8].

Radiological characteristics were as follows: the most common sinuses involved were maxillary (96.43\%), anterior ethmoid and sphenoid (75\%), frontal (71.43\%), and posterior ethmoid (64.29\%). We could not find a research study investigating the types of sinuses involved, and most of them compared radiological findings in terms of unilateral vs bilateral involvement. In this research, bilateral involvement was found in $17(60.71 \%)$ vs unilateral $11(39.29 \%)$. In Makkah in a study conducted in 2017, they found bilateral involvement in $36(69.2 \%)$ vs unilateral 16 (30.8\%) [11].

We found in our research that $13(46.43 \%)$ patients had recurrence symptoms during their follow-up. However, 8 (28.57\%) patients did CT scan which was positive for heterogenous opacification. In contrast to multiple studies, in 
TABLE 5: Recurrence rate and characteristics.

\begin{tabular}{|c|c|c|c|c|}
\hline & & & $N$ & $\%$ \\
\hline \multirow{17}{*}{ Recurrence } & & & 8 & 28.57 \\
\hline & Duration since surgery (month) & & $2.5-27.6$ & 14.9 (mean) \\
\hline & \multirow{2}{*}{ Gender } & Male & 4 & 50 \\
\hline & & Female & 4 & 50 \\
\hline & \multirow{10}{*}{ Clinical presentation } & Nasal obstruction & 7 & 87.5 \\
\hline & & Nasal discharge & 4 & 50 \\
\hline & & PND & 1 & 12.5 \\
\hline & & Facial pressure/pain & 4 & 50 \\
\hline & & Headache & 4 & 50 \\
\hline & & Nasal polyp & 7 & 87.5 \\
\hline & & DNS & 0 & 0 \\
\hline & & HIT & 0 & 0 \\
\hline & & Proptosis & 0 & 0 \\
\hline & & Decreased vision & 0 & 0 \\
\hline & \multirow{3}{*}{ Radiological recurrence } & Unilateral & 2 & 25 \\
\hline & & Bilateral & 6 & 75 \\
\hline & & Uninvolved side previously & 3 & 37.5 \\
\hline
\end{tabular}

TABLE 6: Disease recurrence pattern side.

\begin{tabular}{lccc}
\hline Previous side involved & Recurrence side & $n$ & \% \\
\hline & & 11 & 39.29 \\
Unilateral & Ipsilateral & 2 & 18.18 \\
& Bilateral & 3 & 27.27 \\
Bilateral & Contralateral & 0 & 0 \\
& Bilateral & 17 & 60.71 \\
\hline
\end{tabular}

the eastern province, they found that $54.5 \%$ of patients had recurrence, and in Makkah, it was 55\% [7, 11]. In addition, we studied the pattern of recurrence and if the previously noninvolved side of the sinuses were involved during the recurrence phase. The incidence of ipsilateral recurrence was $2(18.18 \%)$, and the bilateral recurrence was $3(27.27 \%)$ out of the previously affected unilateral side. 3 (17.65) patients out of bilateral AFS patients who were previously diseased had recurrence. However, in a Makkah study, they found that ipsilateral recurrence $12.5 \%$, contralateral $31.3 \%$, and $61.1 \%$ of bilateral AFS patients had recurrence [11].

\section{Conclusion}

A high recurrence rate is a pathognomonic feature of the disease, despite all the development in medical and surgical trials. In addition, more studies with more number of patients are needed in the future to clearly recognize the pattern of recurrence in patients with AFRS.

\section{Data Availability}

The data used to support the findings of this study are included within the article.

\section{Conflicts of Interest}

The authors declare that they have no conflicts of interest.

\section{References}

[1] M. S. Schubert, "Allergic fungal sinusitis," Drugs, vol. 64, no. 4, pp. 363-374, 2004.

[2] A. Vashishth, "Extensive allergic fungal rhinosinusitis: ophthalmic and skull base complications," Indian Journal of Otolaryngology and Head \& Neck Surgery, vol. 67, no. 3, pp. 227-233, 2014.

[3] B. F. Marple, "Allergic fungal rhinosinusitis: a review of clinical manifestations and current treatment strategies," Medical Mycology, vol. 44, no. 1, pp. 277-284, 2006.

[4] J. Rodrigues, C. Caruthers, R. Azmeh, M. S. Dykewicz, R. G. Slavin, and A. P. Knutsen, "The spectrum of allergic fungal diseases of the upper and lower airways," Expert Review of Clinical Immunology, vol. 12, no. 5, pp. 531-550, 2016.

[5] P. Karthikeyan and V. Nirmal Coumare, "Incidence and presentation of fungal sinusitis in patient diagnosed with chronic rhinosinusitis," Indian Journal of Otolaryngology and Head \& Neck Surgery, vol. 62, no. 4, pp. 381-385, 2010.

[6] M. S. Schubert, "Allergic fungal sinusitis: pathophysiology, diagnosis and management," Medical Mycology, vol. 47, no. 1, pp. S324-S330, 2009.

[7] L. M. Telmesani, "Prevalence of allergic fungal sinusitis among patients with nasal polyps," Annals of Saudi Medicine, vol. 29, no. 3, pp. 212-214, 2009.

[8] R. Kaur, S. Lavanya, N. Khurana, A. Gulati, and M. S. Dhakad, "Allergic fungal rhinosinusitis: a study in a tertiary care hospital in India," Journal of Allergy, vol. 2016, p. 7698173, 2016. 
[9] K. T. Montone, V. A. Livolsi, M. D. Feldman et al., "Fungal rhinosinusitis: a retrospective microbiologic and pathologic review of 400 patients at a single university medical center," International Journal of Otolaryngology, vol. 2012, p. 9, 2012.

[10] N. Jamil, "Allergic fungal sinusitis in children in Saudi Arabia," Saudi Medical Journal, vol. 28, pp. 1711-1714, 2007.

[11] O. A. Marglani, R. Kamel, I. R. Herzallah, and A. M. Shaikhb, "Contralateral outcome in unilateral allergic fungal rhinosinusitis: impact on patient management and counseling," Pan Arab Journal of Rhinology Journal, vol. 7, pp. 1-6, 2017. 